Contents List available at VOLKSON PRESS
Multidisciplinary Inclusive Education, Management
and Legal Services (MIEMLS)
Journal Homepage: http://topicsonsocialdevelop.com
DOI: $10.26480 /$ ismiemls.01.2018.04.06

\title{
TRAVEL MODE CHOICE OF LONG-DISTANCE PASSENGERS
}

\author{
Xiangyu Jin1, Chao Wang2*, Wenli Hu ${ }^{3}$ \\ ${ }^{1}$ Institute of logistics management and engineering, Zhuhai College of Jilin University, Zhuhai, China \\ ${ }^{2}$ Department of Library, Zhuhai College of Jilin University, Zhuhai, China \\ ${ }^{3}$ College of accounting and finance, Guangdong Polytechnic of Science and Technology, Zhuhai, China \\ *Corresponding Author Email: yhjxy_2006@126.com
}

This is an open access article distributed under the Creative Commons Attribution License, which permits unrestricted use, distribution, and reproduction in any medium, provided the original work is properly cited.

\section{ARTICLE DETAILS}

\section{Article History:}

Received 26 June 2018

Accepted 2 July 2018

Available online 1 August 2018

\section{ABSTRACT}

This article analyzes the travel mode choice of long distance passengers. Several typical factors influencing the behavior of travel mode choice are put forward, such as generalized time cost, general transportation cost, security, success rate for buying tickets etc. Grouped income level of travelers is introduced into the developed logit model based on theory of utility maximization. Through an empirical investigation, we explore the reasonable strategies for different transportation modes.

\section{KEYWORDS}

Travel mode choice, division model, logit model, theory of utility.

\section{INTRODUCTION}

Owing to the first high-speed railway operated in 2008, competition between civil aviation and high-speed railways become more fiercely, especially in the medium to long distance market. Since passengers have more chances to choose a prefer travel mode, competition relationship between different travel mode has been a popular topic. Especially in recent years, it has been widely studied.

Visitors travel mode selection method based on travel cost was proposed and tested by Saad [1]. Chaug-Ing Hsu proposed a model to analyze market share distribution between high-speed and conventional rail services in a transportation corridor [2]. Work of studying disaggregate travel demand models has taken place since the 1960s', Richard Barff et al. mentioned the majority of research on modeling the mode-choice process [3]. Many models are available to handle mode-choice problems, but three have dominated in this literature: loigit models, probit models and discriminant models. Logit models have been widely applied, ease of estimation is the reason. But its character of IIA makes it limited in application. Numerous efforts have tried to improve the model [3-5]. Lots of scholars put forward a series of improvements on logit model, such as NL model, GNL, photo, etc $[4,6]$. However, the above models were too difficult to solve, and did not fully consider the practical reality decision-making factors.

With the rapid development of high-speed railways, there is a more concern over the competitive relationship between civil aviation and highspeed railways. In this paper, we present an improved logit model to estimate market share distribution in an individual behavior point of view. Our subsequent discussions focus on the following aspects:

1) Updating the conception and algorithm for the factors that affect passengers' traffic choice decision. Introduce revenue into the factors by calculating them in grades.

2) Improving the utility functions and logit model to make the result closer to reality.

3) Parameters of the model are calculated by using maximum likelihood estimation.
The high-speed rail contributes a new option to people, and also affects the structure of passenger transport. Study on the competitive relationship between high-speed railway and air transport, in order to realize the win-win cooperation between them and build a reasonable allocation of resources.

\section{FOUNDATION OF MODEL}

Improved model of travel choice in this paper is based on the theory of utility maximization. This theory has a basic assumption: Passengers are "rational economic man", which means the travelers under certain constraints such as social conditions, economic conditions etc., always choose to make their own utility maximized. We specify foundational model as follows:

$$
U i g=V i g+E i g
$$

As is shown in equation (1), Uig is the total utility when decision maker g choose option i; Vig means the observable utility when decision maker g choose option i; Eig is random disturbance when $g$ choose i.

$$
V_{i g}=\sum_{=1}^{n i} \theta_{i g} X_{i g}
$$

Xig refers to the observable characteristics cause g to select and $\theta i g$ means parameter of observable utility when g choose i. According to theory of utility maximization, travelers will choose travel mode i if the utility of mode $i$ is greater than mode $j$. The formula can be expressed as(3).

$$
V_{i g}+\varepsilon_{i g}>V_{j g}+\varepsilon_{j g}
$$

Then we can obtain Pig , the probability when passenger g choose travel mode $i$, which is presented as (4).

$P_{i g}=P\left\{\varepsilon_{j g} \leq \varepsilon_{i g}+\left(V_{g}-V_{j g}\right)\right\}$

The general form of (4) can be expressed as (5). The value of $k$ ranges from 1 to $n$. 


$$
P_{i g}=\frac{\exp \left[U_{i g}\right]}{\operatorname{Eexp}\left[U_{k g}\right]}
$$

The logit model based on utility maximization theory has caused more attention in area of travel mode choice. We will establish a developed model in the next section.

\section{MODEL DEVELOPMENT}

\subsection{Notations}

Before establishment of the developed logit model, related notations are defined as follows.

$V_{k}$ observable utility of travel mode $\mathrm{k}$

$T_{k}$ generalized travel time of travel mode $\mathrm{k}$

$t_{k 1}$ one-way travel time of mode $\mathrm{k}$

$t_{k 2}$ city travel time of mode $\mathrm{k}$

$t_{k 3}$ delay time of mode i (time of going in and out the station)

$w_{k}$ delay rate of mode $\mathrm{k}$

$C_{k}$ generalized travel cost of travel mode $\mathrm{k}$

$A_{k}$ security of mode k

$S_{k}$ accident rate of mode $\mathrm{k}$

$L_{k}$ successful rate of buying ticket for mode $\mathrm{k}$

$a_{k} 、 b_{k}$ parameters to be estimated

$\varphi_{h}$ time value coefficient

$\overline{G D P_{h}}$ per capita gross national product for city $\mathrm{h}$

$D$ number of working days for one year (Data based on 2015)

\subsection{Influencing Factors Analysis}

Numerous factors affect the travel mode choice of passengers, their behavior may be influenced by several internal and external factors.

1) Internal elements include the individual preference, such as personalities, wage level and travel purpose of passengers.

2) External items relate to the characteristics of different transport modes, such as safety, economy, speed, etc.

According to the data based on survey in this article, age, sex, occupation elements have few effects on travel choice, so we do not choose these items in this paper. The following factors mainly take into account.

\subsubsection{Generalized travel time}

We use the generalized travel time rather than one-way trip time. General travel time includes one-way travel time, city travel time and delay time for different mode choice, which is shown in (6).

$$
T_{i}=\frac{t_{i 1}+t_{i 2}+t_{i 3}}{w_{i}}
$$

\subsubsection{Travel cost}

$C i$ is the travel cost of mode, based on one-way ticket price which airfare includes fuel surcharge. City travel cost can be ignored in this paper because we focus on the long-distance travel.

\subsubsection{Security}

Security is one of the most important factors in mode choice. It is inversely proportional to the accident rate, which is presented in (7). We use the accident statistics' data since 2008, when the first high-speed railway was opened to traffic.

$$
A_{i}=\frac{1}{1+S_{i}}
$$

In addition, we introduce the success rate of buying ticket $L i$ as a significant influencing factor. Because passengers will choose another travel mode while they fail or have difficult to buy ticket.

\subsection{Improved Model}

Previous literature often use "additive model" based on time, cost et al. However, some influencing factors, such as security, its relationship with the final utility is not simple addition. When travelers consider a kind of mode is not safe enough, the probability of choosing this mode will decline exponentially. Therefore, the problem is formulated as a "division model", shown in (8).

$$
V_{k}=\frac{a_{k}^{*} C_{k}+b_{k}{ }^{*} T_{k}}{A_{k}^{*} L_{k}}
$$

Owing to the difference of measuring unit between generalized travel time and travel cost, we use time value coefficient $\varphi_{h}$ to convert cost and time into the same unit.

$$
\varphi_{h}=\frac{\overline{G D P_{h}}}{8^{*} D}
$$

\section{EMPIRICAL ANALYSIS}

As discussed above, the factors that affect mode choice of long-distance passenger also include age, gender, income, travel purpose, etc. For instance, lower income passengers are more sensitive with cost rather than travel time. So income of travelers is taken into consideration. The corresponding time value will be different, due to different income. It can be introduced in (9).

We use the actual data of Beijing-Guangzhou route to calculate in this study. For convenience of calculation, we can classify the income level. According to the questionnaire, revenue can be divided into the following grade, which is shown in table 1.

Passengers whose monthly income between three thousand and five thousand are divided into grade $\mathrm{I}_{1}$.

Table 1: Parameter Calbration

\begin{tabular}{|ll|}
\hline Grades & Monthly income level \\
\hline $\mathrm{I}_{1}$ & $0<$ Income $<3000$ \\
$\mathrm{I}_{2}$ & $3000 \leq$ Income $<5000$ \\
$\mathrm{I}_{3}$ & $5000 \leq$ Income $<9000$ \\
$\mathrm{I}_{4}$ & Income $\geq 9000$ \\
\hline
\end{tabular}

From table 1, we classify the income level of passengers travelling through Beijing-Guangzhou route into four grades. Travelers for different income level have diverse select probability of transportation modes, which can be seen in table 2 .

Table 2: Travel Mode Choice Ratio (\%)

\begin{tabular}{|lllll|}
\hline Grades & \multicolumn{3}{c|}{ Typical Travel modes of long distance passengers } \\
\cline { 2 - 5 } & Aviation & $\begin{array}{l}\text { High-speed } \\
\text { railway }\end{array}$ & $\begin{array}{l}\text { Ordinary } \\
\text { railway }\end{array}$ & Other modes \\
\hline $\mathrm{I}_{1}$ & 0.26 & 0.12 & 91.1 & 4.74 \\
$\mathrm{I}_{2}$ & 20.8 & 10.7 & 66.7 & 1.8 \\
$\mathrm{I}_{3}$ & 40.3 & 21.5 & 28.2 & 1 \\
$\mathrm{I}_{4}$ & 43.5 & 53.5 & 2 & 0.28 \\
\hline
\end{tabular}

According to table 2, we can conclude that civil aviation, high-speed railway and railway play a significant role in long- distance travel. Owing to the limitation of adjustment space in speed and fare, we will ignore common railway in this paper and focus on the study of high-speed rail and air.

\subsection{Parameter Estimation}

As we can see from table 1, traveler can be divided into four types according to income level. For each type, we give the same weight to travel 
time and cost. We introduce the different income grade into formula (4) separately to calculate the time value coefficient. Then the maximum likelihood estimation is used to obtain the parameter estimation results in table 3 . We ignore the grade $\mathrm{I}_{1}$ due to the low probability of choosing highspeed and air.

Table 3: Parameter Calibration

\begin{tabular}{|llll|}
\hline Grades & Parameters & \\
\cline { 2 - 4 } & $\mathrm{a}$ & $\mathrm{b}$ & $\varepsilon$ \\
\hline $\mathrm{I}_{2}$ & -0.0506 & -0.043 & $2.98 \mathrm{E}-10$ \\
$\mathrm{I}_{3}$ & -0.0473 & -0.126 & $1.13 \mathrm{E}-06$ \\
$\mathrm{I}_{4}$ & -0.0163 & -0.323 & $1.78 \mathrm{E}-05$ \\
\hline
\end{tabular}

It can be seen in table3, passengers for different income level have different consideration of travel time and cost. With the increasing of income level, influence of travel time on passengers has increased, but the sensitivity of the generalized cost presents a downward trend. This phenomenon means that travelers with higher revenue have more emphasis on time, but little attention to travel cost.

\subsection{Sensitivity analysis}

This article will discuss how the share rate of high-speed railway and aviation changes, when several factors are altered.

\subsubsection{Changing the ticket price}

Inputting parameters into logit model, the share rate of high-speed rail and aviation can be obtained. Making a little change in ticket price: high-speed railway decreases the ticket price by $10 \%$, the share rate increases about $0.3 \%$; And air fares reduced by $10 \%$, share rate rise about $0.7 \%$. The change performs more significant for travelers belonging to income grade of $\mathrm{I}_{2}$ and $\mathrm{I}_{3}$.

\subsubsection{Changing the ticket price}

Statistic show that successful rate of buying high-speed railway's ticket is about $88 \%$, and the aviation is about $98 \%$. If the rate of high-speed railway rises to $98 \%$, probability of buying railway ticket will increase nearly $5 \%$ for passengers in grade $\mathrm{I}_{4}$. It means that improving the rate of high-speed railway is an important measure to promote the share rate.

\section{CONCLUSION AND FUTURE RESEARCH}

This paper presents an improved logit model to estimate market share distribution in an individual behavior point of view. We are updating the conception and algorithm for the factors that affect passengers' traffic choice decision. Introducing revenue into the factors by calculating them in grades. Improving the utility functions and logit model to make the result closer to reality. Analyzing how share rate between high-speed and aviation altered, when changing the influencing factors. Conclusions based on empirical data are obtained as follows:

Passengers with different income have different considerations in travel mode choice. With the increasing of income level, the influence of travel time on passengers has increased, but the sensitivity of the generalized cost presents the downward trend. Therefore, different marketing strategies can be taken in airlines and railway departments according to different income range of passengers. For instance, railway departments can increase high-speed railway service at night, in response to high income group which prefers time saving, etc. For high-speed railway, improving the success rate of buying tickets is more critical than reduce price. It calls the railway departments to update the web server, simplified buying process and broaden the way of buying tickets to enhance competitiveness. For airlines, ticket discounts is a vital measure to improve its share rate. For cost sensitive travelers, airlines could carry on promotion irregularly. But it needs to discount according to the different time, travel distribution and revenue of passengers.

In this study, graded income is taken into consideration as an important influencing factor. In the future research, personality of passengers, traveller's preference and policy factors will take into account. Furthermore, the article estimates market share distribution in an individual behavior point of view, which will be extended to aggregate level.

\section{ACKNOWLEDGMENTS}

This paper is supported by the project fund: 2017 youth innovation talents project of Guangdong education department (2017GWQNCX015)

\section{REFERENCES}

[1] Saad, N., Aljarad, Black, W.R. 1995. Modeling Saudi Ara-bia-Bahrain Corridor Mode Choice. Journal of Transport Geography, 3 (4), 257-268.

[2] Hsu, C.I., Chung, W.M. 1997. A model for market share distribution between high-speed and conventional rail services in a transportation corridor. The Annals of Regional Science, 31 (2), 121-153.

[3] Petersen, E.R. 2002. A highway corridor planning model. Transportation Research Part A, 36 (2), 107-125.

[4] Piet, R., Vanessa, D. 2004. Determinants of bicycle use: do municipal policies matter. Transportation Research Part A: Policy and Practice, 38 (7), 531-550.

[6] Juan, L., Jinxian, W., Chunfu, S., Hongwei, G. 2016. Cluster-Based Logistic Regression Model for Holiday Travel Mode Choice, In Green Intelligent Transportation System and Safety. Procedia Engineering, 137, 729-737.

[5] Yannis, T., Constantions, A. 2008. Public transit user satisfaction: Variability and policy implications. Transport Policy, 15 (7), 197-208. 